\title{
Influence of Optical Feedback on the Polarization Switching of Vertical-Cavity Surface-Emitting Lasers
}

\author{
C. Masoller and M. S. Torre
}

\begin{abstract}
We study theoretically the effect of isotropic and polarization-selective optical feedback on the polarization switching of vertical-cavity surface-emitting lasers. We use the framework of the spin-flip model that takes into account the spin degree of freedom of carriers in the semiconductor quantum well. We analyze the polarization switching dynamics for different values of the spin relaxation rate and find a good agreement with recent experimental results [5]. We also study the influence of the external cavity length and find different dynamical behaviors for short and long external cavities.
\end{abstract}

Index Terms-laser diodes, optical feedback, polarization control, polarization switching, semiconductor lasers, vertical-cavity surface-emitting lasers (VCSELs).

\section{INTRODUCTION}

V ERTICAL-cavity surface-emitting lasers (VCSELs) are attractive sources for high bit rate optical data transmission. The advantages of VCSELs over conventional, edge-emitting semiconductor lasers are single longitudinal mode operation, low threshold, high modulation efficiency, dense packing capability, and narrow circular beam profile [1].

A drawback of VCSELs is their polarization instability. The output of a VCSEL is usually linearly polarized along one of two orthogonal directions associated with crystalline or stress orientations. When the VCSEL begins to lase, one linear polarization dominates and when the injection current is increased, in many devices it is observed that the emission switches to the orthogonal linear polarization. If the injection current is increased further, higher-order transverse modes are excited, usually with a polarization that is orthogonal to the polarization of the fundamental mode.

The influence of isotropic and polarization-selective optical feedback on the polarization switching of the fundamental transverse mode of VCSELs has been recently studied [2]-[5]. While isotropic feedback induces "channelled behavior" (multiple polarization switching points, see also [6] and [7]) and polarization mode hopping [2], [3], strong enough polarization-selective feedback suppresses the polarization switching, and can be used to obtain polarization-stable emission over the entire range

Manuscript received November 8, 2004; revised December 21, 2004. The work or M. S. Toree was supported in part by a grant from Secretaría de Ciencia y Técnica, (UNCPBA-Argentina) and FONCyT Grant 3/9598.

C. Masoller is with the Departament de Fisica i Enginyeria Nuclear, Universitat Politecnica de Catalunya, E-08222 Terrassa, Spain. He is also with the Instituto de Física, Facultad de Ciencias, Universidad de la República, Montevideo 11400, Uruguay (e-mail: Cristina.masoller@upc.edu).

M. S. Torre is with the Instituto de Física Arroyo Seco, Universidad Nacional del Centro de la Provincia de Buenos Aires, 7000 Tandil, Argentina (e-mail: marita@exa.unicen.edu.ar).

Digital Object Identifier 10.1109/JQE.2005.843923 of injection currents [5]. In [5], Hong et al. interpreted the suppression of the polarization switching as caused by feedback-induced changes of the difference in the gain-to-loss ratio of the two linearly polarized modes (i.e., selective optical feedback changes the effective dichroism). In a polarization switching from one mode $(X-)$ to the orthogonal mode $(Y-), x$-polarized optical feedback favors the $x$-polarized mode, and for increasing feedback it was observed that the polarization switching moves to a higher bias current until it disappears for strong enough feedback; $y$-polarized optical feedback favors the $y$-polarized mode, and with increasing feedback it was observed that the polarization switching moves to a lower bias current, until it disappears.

In this paper, we present results of simulations that are in good agreement with the observations of Hong et al. [5]. We employ a model that is an extension of the well-known spin-flip model proposed by San Miguel et al. [8], and that takes into accounts optical feedback by assuming a single reflection in the external cavity (multiple reflections are neglected and therefore the model is valid for weak and moderate feedback strengths). The thermally induced bandgap red-shift and spatial effects, such as spatial hole burning, which play important roles in the polarization switching dynamics [9]-[12], are also not taken into account. In spite of the simplifications of the model, we find a good qualitative agreement with the experimental findings. A key parameter of the model, that gives rise to different polarization behaviors, is the spin-flip relaxation rate. We investigate the polarization dynamics for various values of the spin-flip rate and find the best agreement with the observations of Hong et al. [5] with a large spin-flip rate. We also study the influence of the external cavity length and find different polarization behaviors for short and long external cavities.

This paper is organized as follows. The model is described in Section II. Section III presents the results of the numerical simulations with different values of the spin relaxation rate, for isotropic and linearly-polarized optical feedback. Section IV contains a summary and the conclusions.

\section{THE MODEL}

The spin-flip model takes into account the spin sublevels of the conduction band and the valence band by considering the amplitudes of the orthogonal circular polarizations $E_{ \pm}$which are associated with transitions between different spin sublevels. $E_{ \pm}$are coupled with two different carrier populations, one with positive, $N_{+}$, and another with negative $N_{-}$spin value. This coupling scheme can be re-written in terms of two orthogonal linearly polarized components $E_{x}$ and $E_{y}$ which are coupled through two carrier populations: the total carrier density $N=N_{+}+N_{-}$and the difference between the carrier 
densities with opposite spin values $n=N_{+}-N_{-}$. In the absence of anisotropies between the two linear polarizations the stationary solutions of the model are linearly polarized states with arbitrary polarization direction [8]. In the presence of anisotropies the direction of the polarization is no longer arbitrary and the stationary solutions are two orthogonal linearly polarized states. The gain difference between the two states, the birefringence and saturable dispersion of the material determine the stability of these states. A linear stability analysis predicts the polarization switching phenomenon for increasing injection current [13].

We extend the spin-flip model to account for weak optical feedback from an external reflector. The solitary VCSEL is assumed to operate in a single longitudinal mode with the fundamental transverse profile. The rate equations for the $x$ - and $y$-linearly polarized slowly-varying complex amplitudes $E_{x}$ and $E_{y}$, the total carrier density $N$, and the population difference $n$ are [8], [13], [14]

$$
\begin{aligned}
\frac{d E_{x}}{d t}= & k(1+i \alpha)\left[(N-1) E_{x}+\operatorname{in} E_{y}\right]-\left(\gamma_{a}+i \gamma_{p}\right) E_{x} \\
& +\eta_{x} E_{x}(t-\tau) \exp (-i \omega \tau)+\sqrt{\beta_{\mathrm{sp}}} \xi_{x}, \\
\frac{d E_{y}}{d t}= & k(1+i \alpha)\left[(N-1) E_{y}-\operatorname{in} E_{x}\right]+\left(\gamma_{a}+i \gamma_{p}\right) E_{y} \\
& +\eta_{y} E_{y}(t-\tau) \exp (-i \omega \tau)+\sqrt{\beta_{\mathrm{sp}}} \xi_{y}, \\
\frac{d N}{d t}= & \gamma_{N}\left[\mu-N\left(1+\left|E_{x}\right|^{2}+\left|E_{y}\right|^{2}\right)\right. \\
& \left.-\operatorname{in}\left(E_{y} E_{x}^{*}-E_{x} E_{y}^{*}\right)\right], \\
\frac{d n}{d t}= & -\gamma_{s} n-\gamma_{N}\left[n\left(\left|E_{x}\right|^{2}+\left|E_{y}\right|^{2}\right)\right. \\
& \left.+i N\left(E_{y} E_{x}^{*}-E_{x} E_{y}^{*}\right)\right] .
\end{aligned}
$$

Here, $k$ is the field decay rate, $\gamma_{N}$ is the decay rate of the total carrier population, $\gamma_{s}$ is the spin-flip rate (which accounts for the mixing of carrier populations with different spins), $\alpha$ the linewidth enhancement factor, $\mu=J / J_{\text {th }}$ where $J$ is the injection current and $J_{\mathrm{th}}$ is the solitary laser threshold current, $\gamma_{a}$ and $\gamma_{p}$ are linear anisotropies representing dicroism and birefringence (positive $\gamma_{a}$ gives the $y$-polarized mode a lower threshold, and positive $\gamma_{p}$ gives the $x$-polarized mode a lower frequency). $\omega$ is the optical frequency of the $x$ - and $y$-polarized modes at the solitary laser threshold in the absence of anisotropies. We take into account that the wavelength of the $x$ - and $y$-modes red-shift with increasing injection current due to device self-heating by considering a linear dependence of $\omega$ with $J$

$$
\omega=\omega_{\mathrm{th}}-2 \pi A\left(J-J_{\mathrm{th}}\right)
$$

where $\omega_{\text {th }}$ is the frequency of the $x$ - and $y$-modes at threshold in the absence of birefringence and $A$ is a constant coefficient. The optical frequencies of the two polarizations $\omega_{x}$ and $\omega_{y}$ vary in the same way with the injection current, and therefore the difference $\omega_{y}-\omega_{x}=\gamma_{p} / \pi$ is independent of the injection.

The optical feedback from the external cavity is represented by the feedback strength into the $x$-polarized mode $\eta_{x}$, the feedback strength into the $y$-polarized mode $\eta_{y}$, and the delay time in the external cavity $\tau$. The last term in (1) and (2) represents the contribution of spontaneous emission noise: $\xi_{x, y}$ are independent Gaussian white noise sources with zero mean and unit variance, and $\beta_{\mathrm{sp}}$ is the noise strength.
The model has steady-state solutions corresponding to linearly polarized states. These are

$$
\begin{aligned}
& E_{x}=\mathcal{E}_{x} e^{i\left(\omega_{x}-\omega\right) t} \quad E_{y}=0 \quad N=\mathcal{N}_{x} \quad n=0 \\
& E_{x}=0 \quad E_{y}=\mathcal{E}_{y} e^{i\left(\omega_{y}-\omega\right) t} \quad N=\mathcal{N}_{y} \quad n=0
\end{aligned}
$$

where

$$
\begin{aligned}
\omega_{x}-\omega & =\alpha\left(\gamma_{a}-\eta_{x} \cos \omega_{x} \tau\right)-\left(\gamma_{p}+\eta_{x} \sin \omega_{x} \tau\right) \\
\omega_{y}-\omega & =-\alpha\left(\gamma_{a}+\eta_{y} \cos \omega_{y} \tau\right)+\left(\gamma_{p}-\eta_{y} \sin \omega_{y} \tau\right) \\
\mathcal{N}_{x} & =1+\left(\gamma_{a}-\eta_{x} \cos \omega_{x} \tau\right) / k \\
\mathcal{N}_{y} & =1-\left(\gamma_{a}+\eta_{y} \cos \omega_{y} \tau\right) / k \\
\mathcal{E}_{x} & =\sqrt{\mu / \mathcal{N}_{x}-1} \\
\mathcal{E}_{y} & =\sqrt{\mu / \mathcal{N}_{y}-1}
\end{aligned}
$$

Solutions corresponding to elliptically polarized states also exist, as discussed in [15]. It can be observed that optical feedback changes not only the net gain difference between the $x$ and $y$-polarized states, but also modifies the frequency difference between them. As in the spin-flip model the stability of the linearly polarized states depends not only on their different gain-to-loss ratios, but also on their frequency difference [13], it can be anticipated that the effect of optical feedback, either isotropic or polarization selective, will not be explained simply in terms of a change of the net gain difference between the linearly polarized states.

In the next section, we show that in spite of the severe simplifications of the model (no spatial effects are taken into account, the thermally induced bandgap redshift is neglected, and only a single reflection in the external cavity is considered), the model has the basic ingredients that allow reproducing the experimental observations of Hong et al. [5].

\section{NUMERICAL RESULTS}

We numerically solve the model equations with typical VCSEL parameters: $k=300 \mathrm{~ns}^{-1}, \alpha=3, \gamma_{n}=1 \mathrm{~ns}^{-1}$, $\beta_{\mathrm{sp}}=10^{-5} \mathrm{~ns}^{-1}$, and $A=120 \mathrm{GHz} / \mathrm{mA}$ (corresponding to a shift in wavelength of about $0.3 \mathrm{~nm} / \mathrm{mA}$ ). To model the experimental conditions of [5], we take $\tau=4 \mathrm{~ns}$ (corresponding to a external cavity length of about $60 \mathrm{~cm}$ ) and $\gamma_{p}=157 \mathrm{GHz}$ (corresponding to a frequency difference between the linearly polarized modes of about $50 \mathrm{GHz}$ [16]). In order to assess the influence of the spin degree of freedom we consider three different values of $\gamma_{s}$ corresponding to slow $\left(\gamma_{s}=10 \mathrm{~ns}^{-1}\right)$, intermediate $\left(\gamma_{s}=50 \mathrm{~ns}^{-1}\right)$, and fast $\left(\gamma_{s}=500 \mathrm{~ns}^{-1}\right)$ spin relaxation.

In the experiments of [5], $J_{\mathrm{th}}=1.8 \mathrm{~mA}$ and the injection current was scanned from 0.9 to $4.9 \mathrm{~mA}$ in $1 \mu \mathrm{s}$. This corresponds to increasing the parameter $\mu$ from 0.5 to 2.5 in $1 \mu \mathrm{s}$ ( $\mu$ was increased in steps of $d \mu=0.0044$ to simulate steps of $0.008 \mathrm{~mA}$ in the experiments). In the experiments a polarization switching was observed, in the absence of feedback, when the current was increased above $J_{\mathrm{PS}}=3.17 \mathrm{~mA}\left(\sim 1.7 J_{\mathrm{th}}\right)$. It was observed that the polarization changed from the high-frequency mode (called $X$ in [5]) to the low-frequency mode (called $Y$ ). This type of polarization switching has been referred to as type-I [17], [18] and it has been understood in terms of thermal effects [9]. At threshold, the polarization state with larger gain is selected. 

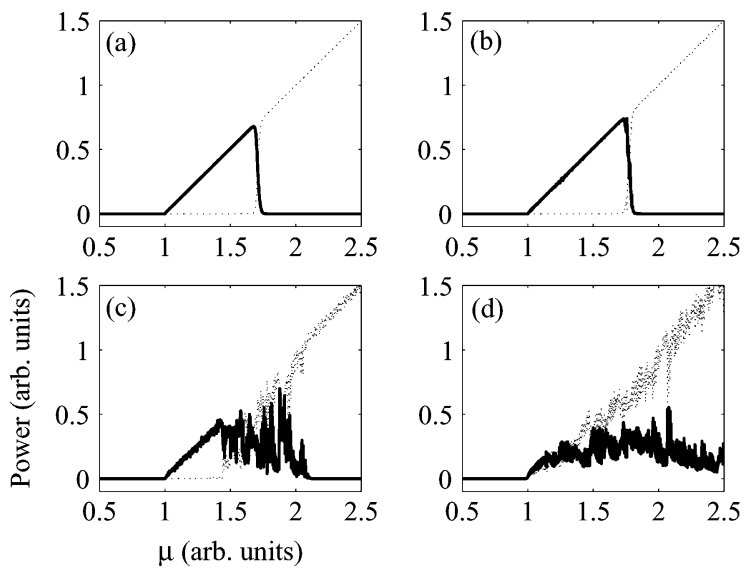

Fig. 1. Polarization-resolved $L-I$ curve for isotropic optical feedback and low spin relaxation rate $\left(\gamma_{s}=10 \mathrm{~ns}^{-1}\right.$ and $\left.\gamma_{a}=1.0 \mathrm{~ns}^{-1}\right)$. (a) $\eta=0.0$. (b) $\eta=1.0 \mathrm{~ns}^{-1}$. (c) $\eta=3.5 \mathrm{~ns}^{-1}$. (d) $\eta=30 \mathrm{~ns}^{-1}$. The solid thick line (dotted thin line) shows the $y-(x-)$ modal intensity.

As the injection current increases, the semiconductor medium heats up due to Joule's effect and, as a consequence, there is a redshift of both the gain spectrum and the cavity resonances. The redshift of the gain spectrum is faster that the redshift of the cavity resonances, and this might lead to a change of sign in the gain difference between the two polarizations and thus to a polarization switching. However, type-I polarization switching has also been observed under pulsed operation, where the temperature of the active region is nearly constant [19], and this suggesst the existence of additional nonthermal mechanisms for polarization switching. We remark that in the framework of the spin-flip model, type I polarization switching has a nonthermal origin and occurs even when $A=0$ [13].

Since in the spin-flip model, if $\gamma_{p}>0$, the $y$-polarized mode is the high-frequency mode, we considered positive $\gamma_{a}$ (which gives the $y$-polarized mode a lower threshold) and adjusted the value of $\gamma_{a}$ such that a polarization switching from the $y$ - to the $x$-polarized mode occurs, in the absence of feedback, at $J_{\mathrm{PS}} / J_{\mathrm{th}}=\mu=1.7$. The larger the value of $\gamma_{s}$, the lower the value of $\gamma_{a}$ needed to observe the polarization switching at $\mu=1.7$. The intensities of the $x$ - and $y$-polarized modes were averaged over a time window of $1 \mathrm{~ns}$ to simulate the bandwidth of the experimental detectors. We present results for different optical feedback strengths. One has to keep in mind when comparing the cases of low and large $\gamma_{s}$ that at the same time they represent situations with large and low dichroism; when comparing the simulations with the experiments, the $y-(x-)$ polarized mode in the equations corresponds to the $x$ - $(y$-) polarized mode in the experiments.

Fig. 1 displays the polarization-resolved $L-I$ curve for isotropic feedback $\left(\eta_{x}=\eta_{y}=\eta\right)$, a low value of the spin relaxation rate, and large dichroism. Fig. 1(a) displays the polarization switching in the absence of feedback (which occurs at about $\mu=1.7$ ), and Fig. 1(b)-(d) displays results for increasing feedback levels. Figs. 2 and 3 display the polarization resolved $L-I$ curve for intermediate and large $\gamma_{s}$. It can be observed that weak isotropic optical feedback perturbs only slightly the solitary laser polarization switching point [Figs. 1(b), 2(b), and 3(b)]. For increasing feedback strength there is a region of values of the injection current where both polarization modes either coexist [Fig. 1(c)] or alternate [Figs. 2(c) and 3(c)].
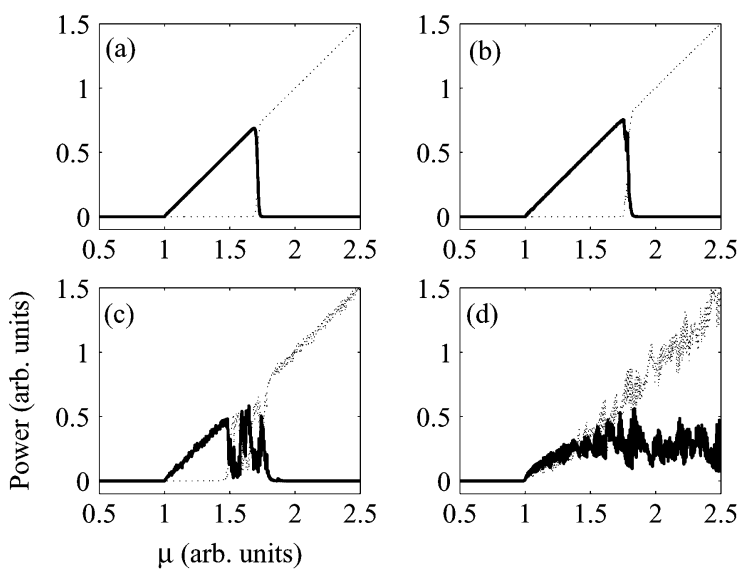

Fig. 2. Polarization-resolved $L-I$ curve for isotropic optical feedback and intermediate spin relaxation rate $\left(\gamma_{s}=50 \mathrm{~ns}^{-1}\right.$ and $\left.\gamma_{a}=0.95 \mathrm{~ns}^{-1}\right)$. (a) $\eta=0.0$. (b) $\eta=1.0 \mathrm{~ns}^{-1}$. (c) $\eta=3 \mathrm{~ns}^{-1}$. (d) $\eta=30 \mathrm{~ns}^{-1}$. The solid thick line (dotted thin line) shows the $y$ - $(x-)$ modal intensity.
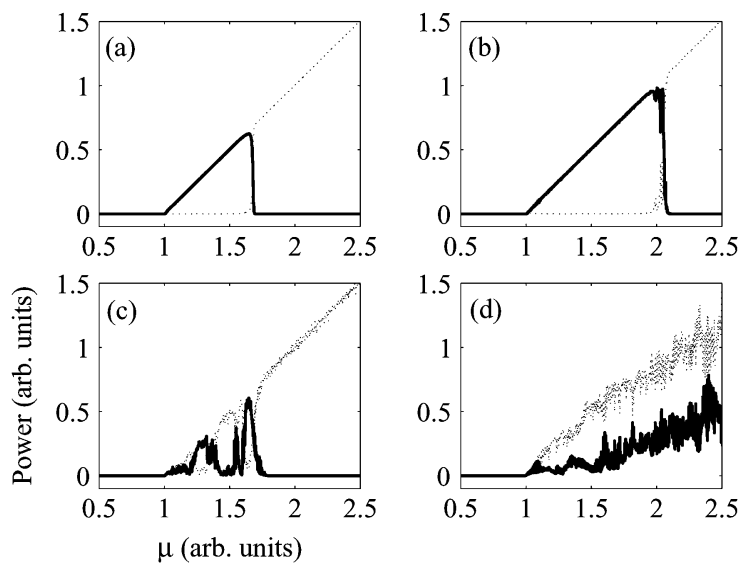

Fig. 3. Polarization-resolved $L-I$ curve for isotropic optical feedback and large spin relaxation rate $\left(\gamma_{s}=500 \mathrm{~ns}^{-1}\right.$ and $\left.\gamma_{a}=0.18 \mathrm{~ns}^{-1}\right)$. (a) $\eta=0.0$. (b) $\eta=1 \mathrm{~ns}^{-1}$. (c) $\eta=2.3 \mathrm{~ns}^{-1}$. (d) $\eta=25 \mathrm{~ns}^{-1}$. The solid thick line (dotted thin line) shows the $y$ - $(x-)$ modal intensity.

For larger feedback strength both polarizations coexist, with the $x$-polarized mode being the dominant one [see Figs. 1(d), $2(d)$, and $3(d)$; a similar behavior has been observed in [20, Fig. 10(a)].

The above scenario agrees qualitatively well with the experimental observations of Hong et al. ([5, Fig. 3]); however, the agreement is better for intermediate and large $\gamma_{s}$ because in these cases there is a range of injection currents where there is well defined "channelled behavior" [see Figs. 2(c) and 3(c) where it can be observed that the polarization alternates as the current increases: one polarization dominates and the other is at the noise level]. This region occurs between the threshold and the solitary laser polarization switching point $(\mu=1.7)$, in agreement with the experiments. In the case of low $\gamma_{s}$, there is no polarization alternation but rather a range of injection currents where both polarizations coexist.

The polarization alternation and the polarization coexistence shown in Figs. 1(c), 2(c), and 3(c) occurs for feedback levels above a certain threshold which depends on the noise strength: if the feedback is weak, by itself it does not induce polarization coexistence or alternation; however, with the inclusion of noise in the simulations polarization instabilities are observed. 

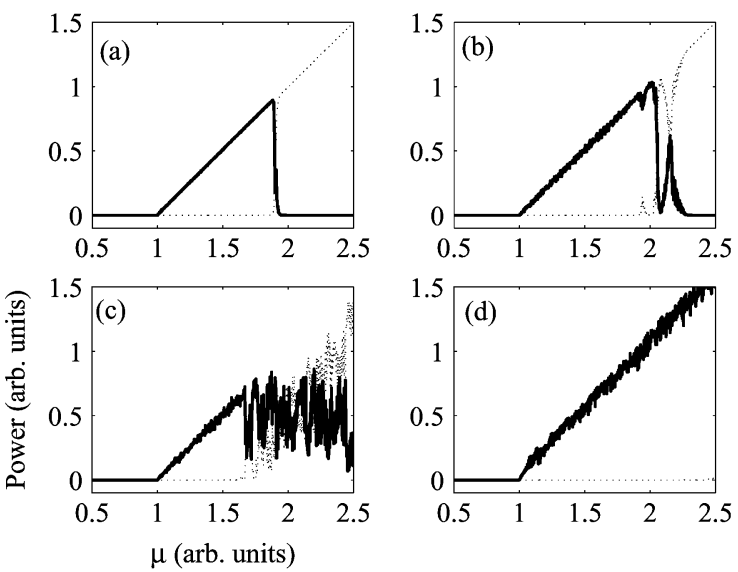

Fig. 4. Polarization-resolved $L-I$ curve for $y$-polarized optical feedback $\left(\eta_{x}=0, \eta_{y}=\eta\right)$ and low spin relaxation rate $\left(\gamma_{s}=10 \mathrm{~ns}^{-1}\right.$, and $\gamma_{a}=1.0$ $\mathrm{ns}^{-1}$ ). (a) $\eta=1$. (b) $\eta=2 \mathrm{~ns}^{-1}$. (c) $\eta=4 \mathrm{~ns}^{-1}$; (d) $\eta=20 \mathrm{~ns}^{-1}$. The solid thick line (dotted thin line) shows the $y$ - $(x-)$ modal intensity.
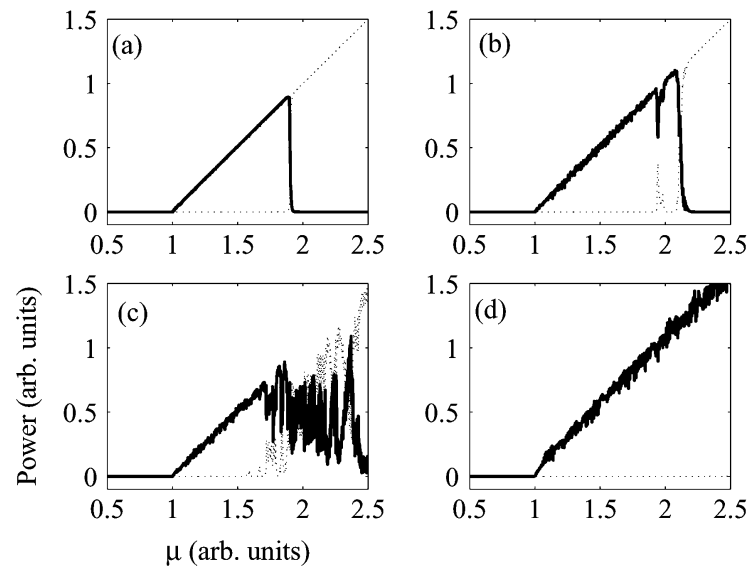

Fig. 5. Polarization-resolved $L-I$ curve for $y$-polarized optical feedback $\left(\eta_{x}=0, \eta_{y}=\eta\right)$ and intermediate spin relaxation rate (parameters as Fig. 2) (a) $\eta=1$. (b) $\eta=2 \mathrm{~ns}^{-1}$. (c) $\eta=3 \mathrm{~ns}^{-1}$. (d) $\eta=20 \mathrm{~ns}^{-1}$. The solid thick line (dotted thin line) shows the $y$ - $(x-)$ modal intensity.

At larger feedback levels, feedback-induced polarization competition occurs without the need of including noise in the simulations.

Let us now present results for polarization-selective feedback. Figs. 4-6 display the polarization-resolved $L-I$ curve for $y$-polarized optical feedback $\left(\eta_{x}=0, \eta_{y}=\eta\right)$ and low, intermediate, and large $\gamma_{s}$, respectively. A good qualitative agreement with Fig. 4 of [5] is observed. In the three cases, for strong enough $y$-polarized feedback the polarization switching phenomenon is suppressed and $y$-polarized emission occurs in the hole range of injection currents. It can be noticed, however, that the transition to polarization-stable emission for large $\gamma_{s}$ is different from that for low and intermediate $\gamma_{s}$. For large $\gamma_{s}$ (and small dichroism), the optical feedback moves the polarization switching point gradually to the left (toward higher injection currents, see Fig. 6). In the cases of low and intermediate $\gamma_{s}$, there is a different scenario. As the feedback increases, the $y$-polarized mode gradually turns on in the region of high injection currents (where it is off in the absence of feedback) and competes with the $x$-polarized mode. As the strength of the feedback increases, the $y$-polarized mode gradually becomes dominant
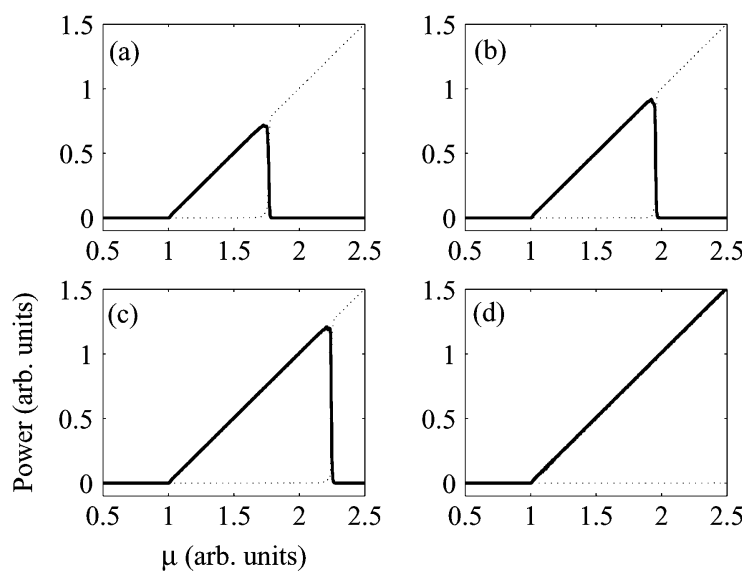

Fig. 6. Polarization-resolved $L-I$ curve for $y$-polarized optical feedback $\left(\eta_{x}=0, \eta_{y}=\eta\right)$ and large spin relaxation rate $\left(\gamma_{s}=500 \mathrm{~ns}^{-1}\right.$ and $\gamma_{a}=0.18 \mathrm{~ns}^{-1}$ ). (a) $\eta=0.1 \mathrm{~ns}^{-1}$. (b) $\eta=0.2 \mathrm{~ns}^{-1}$. (c) $\eta=0.4 \mathrm{~ns}^{-1}$. (d) $\eta=1 \mathrm{~ns}^{-1}$. The solid thick line (dotted thin line) shows the $y$ - $(x-)$ modal intensity.
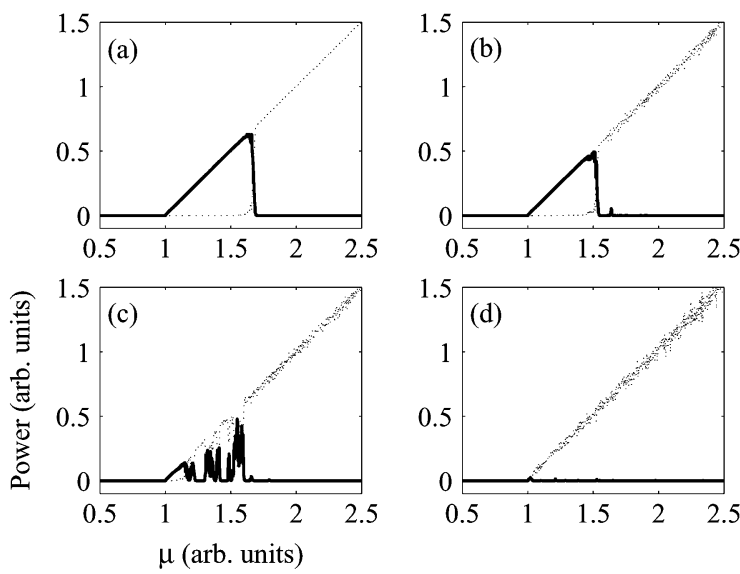

Fig. 7. Polarization-resolved $L-I$ curve for $x$-polarized optical feedback $\left(\eta_{y}=0, \eta_{x}=\eta\right)$ and low spin relaxation rate $\left(\gamma_{s}=10 \mathrm{~ns}^{-1}\right.$ and $\gamma_{a}=1.0$ $\mathrm{ns}^{-1}$ ). (a) $\eta=1.0$. (b) $\eta=2.0 \mathrm{~ns}^{-1}$. (c) $\eta=3 \mathrm{~ns}^{-1}$. (d) $\eta=10 \mathrm{~ns}^{-1}$. The solid thick line (dotted thin line) shows the $y$ - $(x-)$ modal intensity.

and the $x$-polarized mode gradually becomes suppressed until it finally turns off for strong feedback. Figs. 7-9 display the polarization-resolved $L-I$ curve for $x$-polarized optical feedback $\left(\eta_{y}=0, \eta_{x}=\eta\right)$ and low, intermediate, and large $\gamma_{s}$, respectively. These results should be compared with experimental observations with $y$-polarized feedback [5, Fig. 5]. A good qualitative agreement can be observed for strong enough feedback the polarization switching phenomenon is suppressed and $x$-polarized emission is observed in the hole range of injection currents. Again, there is a different transition scenario for large $\gamma_{s}$ and for low and intermediate $\gamma_{s}$. In the first case, for increasing feedback, the polarization switching point moves to the left (toward lower injection currents, see Fig. 9) and abruptly disappears when the feedback strength increases above a certain value. For low and intermediate $\gamma_{s}$, as the feedback increases, the $x$-polarized mode gradually turns on in the region of low injection currents (where it is off in the absence of feedback) and competes with the $y$-polarized mode (Figs. 7 and 8). As the strength of the feedback increases the $x$-polarized mode gradually becomes dominant and the $y$-polarized mode gradually becomes suppressed until finally does not turn at all. 

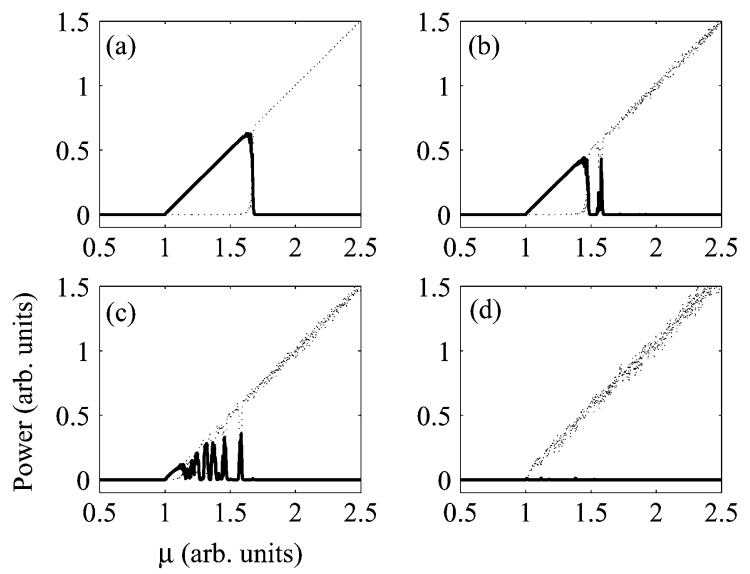

Fig. 8. Polarization-resolved $L-I$ curve for $x$-polarized optical feedback $\left(\eta_{y}=0, \eta_{x}=\eta\right)$ and intermediate spin relaxation rate $\left(\gamma_{s}=50 \mathrm{~ns}^{-1}\right.$ and $\gamma_{a}=0.95 \mathrm{~ns}^{-1}$ ). (a) $\eta=1$. (b) $\eta=2 \mathrm{~ns}^{-1}$. (c) $\eta=3 \mathrm{~ns}^{-1}$. (d) $\eta=20$ $\mathrm{ns}^{-1}$. The solid thick line (dotted thin line) shows the $y$ - $(x-)$ modal intensity.
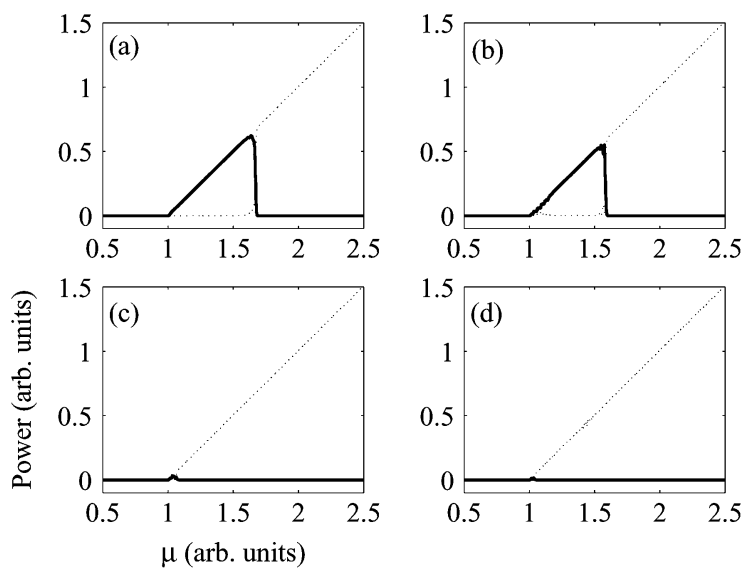

Fig. 9. Polarization-resolved $L-I$ curve for $x$-polarized optical feedback $\left(\eta_{y}=0, \eta_{x}=\eta\right)$ and large spin relaxation rate $\left(\gamma_{s}=500 \mathrm{~ns}^{-1}\right.$ and $\gamma_{a}=0.18 \mathrm{~ns}^{-1}$ ). (a) $\eta=0.1$. (b) $\eta=0.6 \mathrm{~ns}^{-1}$. (c) $\eta=0.65 \mathrm{~ns}^{-1}$. (d) $\eta=1 \mathrm{~ns}^{-1}$. The solid thick line (dotted thin line) shows the $y-(x-)$ modal intensity.

We remark that for low and intermediate $\gamma_{s}$, polarizationstable emission is obtained at the cost of a chaotic output, which exhibits the typical instabilities induced by optical feedback. It can be noticed that for $\gamma_{s}=10$ and $\gamma_{s}=50 \mathrm{~ns}^{-1}$ relatively large feedback strengths are required in order to suppress the polarization switching phenomenon. On the contrary, for large $\gamma_{s}$ weak feedback strengths are able to suppress the polarization switching without inducing a chaotic output. This difference is due to the dichroism: for low and intermediate $\gamma_{s}$, the parameter $\gamma_{a}$ has to be large $\left(\gamma_{a} \sim 1 \mathrm{~ns}^{-1}\right)$ to observe the polarization switching of the solitary laser at $\mu=1$.7, while it has to be lower for large $\gamma_{s}$ (compared with $\gamma_{a} \sim 0.2 \mathrm{~ns}^{-1}$ for $\gamma_{s}=500 \mathrm{~ns}^{-1}$ ). A higher intrinsic dichroism implies that higher polarization-selective feedback levels will be required in order to modify the net gain difference between the two polarization modes.

In the previous results, the real influence of the spin-flip rate is been hidden by the simultaneous variation of the parameter $\gamma_{a}$. To assess the importance of the spin dynamics we now consider the polarization switching that occurs for low and large spin-flip rate, keeping the dichroism parameter $\gamma_{a}$ fixed. Fig. 10(a) and (b) displays the polarization switching of the solitary laser for
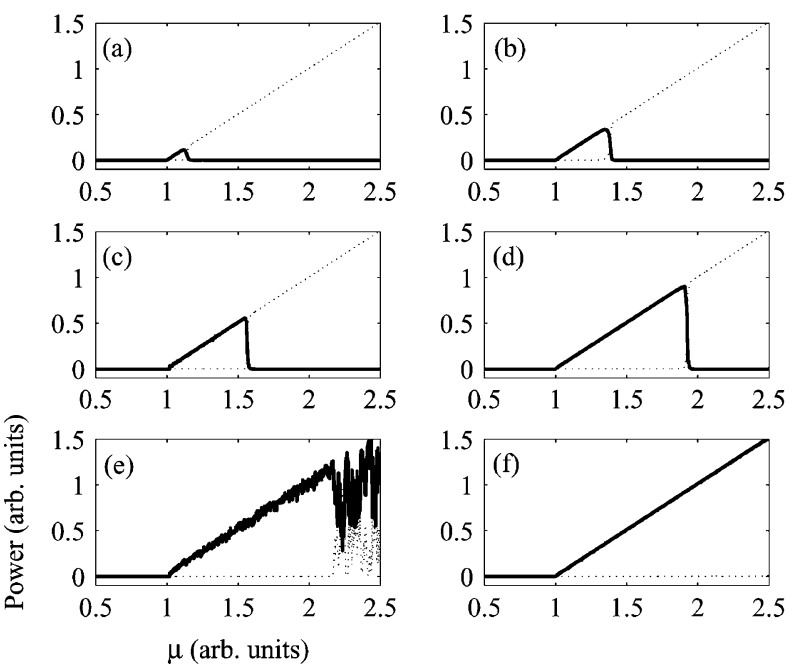

Fig. 10. Influence of the spin-flip rate. (a), (c), (e) $\gamma_{s}=10 \mathrm{~ns}^{-1}$. (b), (d), (f) $\gamma_{s}=500 \mathrm{~ns}^{-1}$. (a)-(b) Polarization-resolved $L-I$ curve of the solitary laser. (c)-(f) Polarization-resolved $L-I$ curve of the laser with $y$-polarized optical feedback. (c) $\eta_{y}=1 \mathrm{~ns}^{-1}$. (d) $\eta_{y}=0.5 \mathrm{~ns}^{-1}$. (e) $\eta_{y}=10 \mathrm{~ns}^{-1}$. (f) $\eta_{y}=1$ $\mathrm{ns}^{-1}$. Other parameters are $\gamma_{a}=0.2 \mathrm{~ns}^{-1}$ and $\gamma_{p}=200 \mathrm{GHz}$.

low and large $\gamma_{s}$ respectively, and Fig. 10(c)-(f) displays the effect of $y$-polarized optical feedback. In spite of the fact that the dichroism is the same, the feedback strength needed to control the polarization for low $\gamma_{s}$ is much larger than for large $\gamma_{s}$ [notice the different feedback levels in Fig. 10(c) and (d), and in Fig. 10(e) and (f)]. In Fig. 10(e), it can be observed that optical feedback induces instabilities in the output intensity. On the contrary, in the case of large $\gamma_{s}$, weak $y$-polarized optical feedback is enough to suppress the polarization switching, and in Fig. 10(f) no feedback-induced instabilities are observed. The different feedback strengths needed to suppress the polarization switching are due to the fact that the polarization state of the light emitted by a VCSEL is linked not only to cavity anisotropies (which are modified by selective optical feedback) but also to the field-material physics (i.e., to the angular momentum of the quantum states involved in the transitions for emission and absorption). In the spin-flip model not only the relative strength of the gain of the two linearly polarized states, but also the birefringence and the saturable dispersion of the material determine the stability of these states [13].

So far we have presented results for a fixed delay time ( $\tau=4 \mathrm{~ns}$ ). Let us now study the influence of the delay time (i.e., of the external cavity length) on the control of the polarization switching by use of weak polarization-selective optical feedback. First, we analyze the effect of $\tau$ in the case of $y$-polarized optical feedback. A summary of results is presented in Fig. 11, where the left column displays results for low $\gamma_{s}$ and the right column for large $\gamma_{s}$. The feedback level is kept constant; only the delay time is varied. For a very short external cavity ( $\tau \ll 1 \mathrm{~ns}$ ), multiple polarization switching points are observed and the behavior is qualitatively the same for low and large $\gamma_{s}$ [Fig. 11(a) and (b)]. This regular switching can be understood in terms of a feedback-induced change of the net gain difference between the two polarizations [6], [7]: the variation of the optical frequencies with the injection (5) leads injection-dependent gains through the dependence of $\mathcal{N}_{x}$ and $\mathcal{N}_{y}$ on $\omega_{x}$ and $\omega_{y}(8)$. The origin of this regular switching 

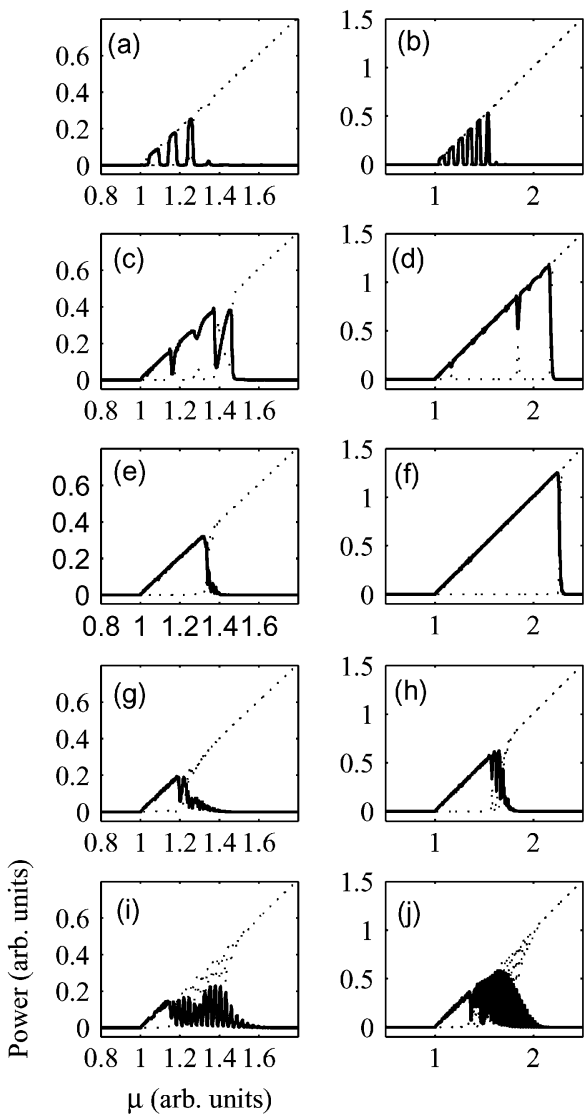

Fig. 11. Influence of the delay time in the case of $y$-polarized optical feedback $\left(\eta_{y}=1 \mathrm{~ns}^{-1}, \eta_{x}=0\right)$. The left column displays results for low spin-flip rate $\left(\gamma_{s}=10 \mathrm{~ns}^{-1}\right)$, and the right column, for large spin-flip rate $\left(\gamma_{s}=500 \mathrm{~ns}^{-1}\right)$. (a)-(b) $\tau=0.05 \mathrm{~ns}$. (c)-(d) $\tau=1 \mathrm{~ns}$. (e)-(f) $\tau=6 \mathrm{~ns}$. (g)-(h) $\tau=11 \mathrm{~ns}$. (i)-(j) $\tau=11.5 \mathrm{~ns}$. Other parameters are as in Fig. 10.

behavior is deterministic but to actually observe the switching as the current increases one must include some noise in the simulations to trigger the switchings.

As the delay increases the effect of the feedback becomes more complex and can not be understood simply in terms of changes of the gain-to-loss ratios. The switchings become more sporadic [Fig. 11(c) and (d)] and for $\tau$ large enough, there is again a single polarization switching point [Fig. 11(e) and (f)]. The value of the injection current for which the switching occurs depends on the delay time and on the feedback strength, as shown in Fig. 12. This suggest that if one does not wish to completely suppress the polarization switching but only to control the polarization state of the light such that there is stable polarization emission in a certain range of injection currents, this can be achieved using $y$-polarized feedback, by adjusting the parameters $\eta$ and $\tau$, such that the polarization switching point moves outside the range of interest.

For long external cavities (roughly speaking, $\tau \geq 10 \mathrm{~ns}$ ), the polarization switching does not occur at a well-defined point, but rather there is an interval of values of $\mu$ where both polarizations co-exist and compete [Fig. 11(g) and (h)]. Moreover, there are values of $\tau$ for which again a multiple polarization switching behavior is observed [Fig. 11(i) and (j)]. However, the time-dependent dynamics is different from that occurring for short delays: for long delays the polarization-resolved intensities exhibit chaotic anti-phase behavior (which is displayed
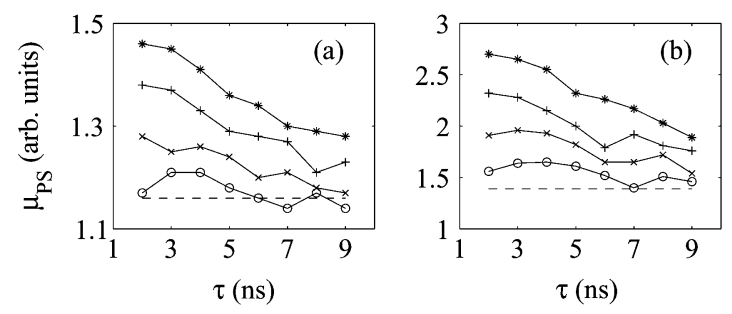

Fig. 12. Polarization switching point as a function of the delay time, in the case of $y$-polarized optical feedback. (a) $\gamma_{s}=10 \mathrm{~ns}^{-1}$. (b) $\gamma_{s}=500 \mathrm{~ns}^{-1}$. $\eta_{y}=0.25 \mathrm{~ns}^{-1}(\mathrm{o}), 0.5 \mathrm{~ns}^{-1}(\times), 0.75 \mathrm{~ns}^{-1}(+), 1.0 \mathrm{~ns}^{-1}(*)$. The dashed line indicates the polarization switching point in the absence of feedback. Other parameters are as in Fig. 10.
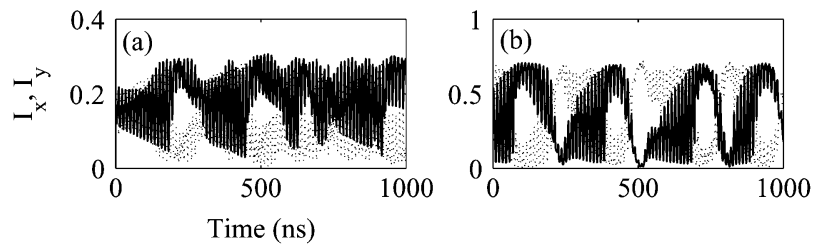

Fig. 13. Polarization-resolved intensity time traces in the case of $y$-polarized optical feedback from a long external cavity. The solid thick line (dotted thin line) shows the $y$ - $(x-)$ modal intensity. The intensities are averaged over 1 ns to simulate the bandwidth of the experimental detectors. (a) $\gamma_{s}=10 \mathrm{~ns}^{-1}, \mu=$

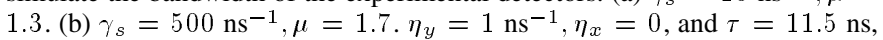
other parameters as in Fig. 10.

in Fig. 13), while for short delays the polarization-resolved intensities are constant in time. Our simulations suggest that the anti-phase dynamics occurring for long-delays has a deterministic origin, but the range of injection currents in which it occurs is enhanced by the inclusion of noise.

The effect of $x$-polarized optical feedback also depends on the delay time. For very short delays multiple regular switchings are observed, while for long delays, depending on the value of $\tau$ either the polarization switching is suppressed, or it moves to lower injection currents, or it is not affected by the feedback.

Finally, let us analyze how these results depend on the value of the parameter $A$ that represents the redshift of the laser wavelength due to device self-heating as the injection current increases. The regular multiple polarization switching points occurring for short delays are due to injection-dependent gains through injection-dependent optical frequencies and therefore do not exist if $A=0$. In the case of longer delays, the parameter $A$ does not modify qualitatively the dynamics, but it changes the feedback level needed for stable polarization emission, or it changes the value of the injection current at which the polarization switching occurs. In particular, the multiple polarization switchings observed for long delays [Fig. 11(i) and (j)] are also observed if $A=0$, however, they are less pronounced and they occur in smaller intervals of the injection current.

\section{SUMARY AND CONCLUSION}

The effect of isotropic and polarization-selective optical feedback on the polarization switching of VCSELs was studied numerically. We analyzed the effect of feedback in three different situations: slow, intermediate, and fast spin relaxation. For slow and intermediate spin relaxation, we considered a rather high dichroism, while for fast spin relaxation we considered a lower dichroism. In the three cases, the parameters were adjusted such that for the solitary laser a polarization 
switching from the high to the low frequency mode occurred at $\mu_{\mathrm{PS}}=1.7$ (corresponding to the experimental situation of [5]). We found a good qualitative agreement with the observations of [5]: strong enough polarized feedback leads to polarization stable emission. The agreement is better for intermediate and high $\gamma_{s}$ as in these cases, for isotropic feedback, channelled behavior with the alternation of the dominant polarization was also clearly observed. We also studied the influence of the external cavity length and found qualitatively different dynamical behaviors for short and long external cavities.

Our results might be of interest for polarization-sensitive optical systems based on VCSELs. For example, if one desires stable $x$-polarized emission over the entire range of injection currents $[0, \mu]$ with $\mu>\mu_{\mathrm{PS}}, x$-polarized feedback can be used just in the transient turn-on, to avoid the initial emission of $y$-polarized light: once the injection current has reached its final value $\mu>\mu_{\mathrm{PS}}$, the $x$-polarized feedback can be turned off.

\section{ACKNOWLEDGMENT}

The authors would like to thank Y. Hong for enlightening discussions.

\section{REFERENCES}

[1] "VCSELs and optical interconnects," in Proc. SPIE, vol. 4942, H. Thienpont and J. Danckaert, Eds., 2003.

[2] M. Sciamanna, K. Panajotov, H. Thienpont, I. Veretennicoff, P. Mégret, and M. Blondel, "Optical feedback induces polarization mode hopping in vertical-cavity surface-emitting lasers," Opt. Lett., vol. 28, pp. $1543-1545,2003$.

[3] K. Panajotov, M. Sciamanna, A. Tabaka, P. Megret, M. Blondel, G. Giacomelli, F. Marin, H. Thienpont, and I. Veretennicoff, "Residence time distribution and coherence resonance of optical-feedback-induced polarization mode hopping in vertical-cavity surface-emitting lasers," Phys. Rev. A, vol. 69, no. $011801,2004$.

[4] K. Panajotov, M. Arizaleta, M. Camarena, H. Thienpont, H. J. Unold, J. M. Ostermann, and R. Michalzik, "Polarization switching induced by phase change in extremely short external cavity vertical-cavity surfaceemitting lasers," Appl. Phys. Lett., vol. 84, pp. 2763-2765, 2004.

[5] Y. Hong, P. S. Spencer, and K. A. Shore, "Suppression of polarization switching in vertical-cavity surface-emitting lasers by use of optical feedback," Opt. Lett., vol. 29, pp. 2151-2153, 2004

[6] A. Valle, L. Pesquera, and K. A. Shore, "Polarization selection and sensitivity of external cavity vertical-cavity surface-emitting laser diodes," IEEE Photon. Tech. Lett., vol. 10, no. 5, pp. 639-641, May 1998.

[7] P. Besnard, M. L. Chares, G. Stephan, and F. Robert, "Switching between polarized modes of a vertical-cavity surface-emitting laser by isotropic optical feedback," J. Opt. Soc. Amer. B., vol. 17, pp. 1057-1063, 1999

[8] M. S. Miguel, Q. Feng, and J. V. Moloney, "Light-polarization dynamics in surface-emitting semiconductor lasers," Phys. Rev. A, Gen. Phys., vol. 52, pp. 1728-1739, 1995

[9] K. D. Choquette, R. P. Schneider Jr., K. L. Lear, and R. E. Leibenguth, "Gain-dependent polarization properties of vertical-cavity lasers," IEEE J. Select. Topics Quantum Electron., vol. 1, no. 2, pp. 661-666, Jun. 1995.

[10] S. Balle, E. Tolkachova, M. S. Miguel, J. R. Tredicce, J. Martin-Regalado, and A. Gahl, "Mechanisms of polarization switching in single-transverse-mode vertical-cavity surface-emitting lasers: Thermal shift and nonlinear semiconductor dynamics," Opt. Lett., vol. 24, pp. $1121-1123,1999$

[11] M. Sondermann, M. Weinkath, T. Ackemann, J. Mulet, and S. Balle, "Two-frequency emission and polarization dynamics at lasing threshold in vertical-cavity surface-emitting lasers," Phys. Rev. A, Gen. Phys., vol. 68 , no. $033822,2003$.
[12] J. Martín-Regalado, S. Balle, M. S. Miguel, A. Valle, and L. Pesquera, "Polarization and transverse-mode selection in quantum-well vertical-cavity surface-emitting lasers: Index- and gain-guided devices," Quantum Semiclass. Opt., vol. 9, pp. 713-736, 1997.

[13] J. Martín-Regalado, F. Prati, M. S. Miguel, and N. B. Abraham, "Polarization properties of vertical-cavity surface-emitting lasers," IEEE J. Quantum Electron., vol. 33, no. 5, pp. 765-783, May 1997.

[14] M. S. Miguel, Semiconductor Quantum Optoelectronics, A. Miller, M. Ebrahimzadeh, and D. M. Finlayson, Eds. Bristol, MA: Inst. Phys., 1999 , p. 339.

[15] C. Masoller and N. B. Abraham, "Low-frequency fluctuations in vertical-cavity surface-emitting semiconductor lasers with optical feedback," Phys. Rev. A, Gen. Phys. , vol. 59, pp. 3021-3031, 1999

[16] Y. Hong, private communication, Nov. 2004.

[17] B. Ryvkin, K. Panajotov, A. Georgievski, J. Danckaert, M. Peeters, G. Verschaffelt, H. Thienpont, and I. Veretennicoff, "Effect of photon-energy-dependent loss and gain mechanisms on polarization switching in vertical-cavity surface-emitting lasers," J. Opt. Soc. Amer. B, vol. 16, pp. 2106-2113, 1999.

[18] T. Ackemann and M. Sondermann, "Characteristics of polarization switching from the low to the high frequency mode in vertical-cavity surface-emitting lasers," Appl. Phys. Lett., vol. 78, pp. 3574-3576, 2001.

[19] J. Martín-Regalado, J. L. A. Chilla, J. J. Rocca, and P. Brusenbach, "Polarization switching in vertical-cavity surface-emitting lasers observed at constant active region temperature," Appl. Phys. Lett., vol. 70, pp. 3350-3352, 1997.

[20] A. V. Naumenko, N. A. Loiko, M. Sondermann, and T. Ackemann, "Description and analysis of low-frequency fluctuations in vertical-cavity surface-emitting lasers with isotropic optical feedback by a distant reflector," Phys. Rev. A, Gen. Phys., vol. 68, no. 033 805, 2003.

C. Masoller was born in Montevideo, Uruguay, in 1963. She received the M.Sc. degree in physics from the Universidad de la Republica, Montevideo, Uruguay, in 1991 and the Ph.D. degree in physics from Bryn Mawr College, PA, in 1999.

Since 1993 she has been with the Physics Department of the School of Sciences, Universidad de la Republica, Uruguay, where she is an Associate Professor. She is also presently at the Departament de Fisica i Enginyeria Nuclear, Universitat Politecnica de Catalunya in Spain, with a "Ramon and Cajal" research position. She was a Visiting Professor at the Physics Department, Universitat de les Illes Balears, Spain (September-October 2001, February-March 2002, and September 2002) and a Visiting Researcher at the School of Informatics, University of Wales, Bangor, U.K. (October 2002-January 2003 and October-November 2003). In 2003, she became a Regular Associate of the Abdus Salam International Centre for Theoretical Physics, Trieste, Italy. She has authored or coauthored over 50 journal papers. She was co-organizer of the "First Rio de la Plata Workshop on Chaos, Noise and Complexity in Lasers and Nonlinear Optics," held in Colonia, Uruguay, in December 2003. Her research interests include theoretical modeling of chaotic systems, synchronization and stochastic phenomena, nonlinear dynamics of semiconductor lasers, and time-delayed systems.

M. S. Torre received the Licenciada en Fisica (Ms.Sc.) and the Ph.D. degrees from the Universidad Nacional del Centro de la Provincia de Buenos Aires (UNCPBA). Her research was primarily in external driven laser physics.

From 1995 to 1997, she was a Postdoctoral Fellow at the Photonics Technology Department, ETSI Telecomunicaciones, Universidad Politécnica de Madrid, Madrid, Spain. Her research was in quantum-well semiconductor-laser modeling. Since 1988 she has been member of the Quantum Electronic Group of the Physics Institute Arroyo Seco. She is currently a Research Professor at the Facultad de Ciencias Exactas of the UNCPBA. Her research topics include modeling and dynamics of vertical-cavity surface-emitting lasers, dynamics of semiconductor laser with external optical feedback, and diffusion effects in semiconductor lasers. 